\title{
Design and Realization of the Safe Driving Speed Analytical System
}

\author{
Liu Bin ${ }^{1, a}$
}

${ }^{1}$ Department of Transportation Engineering, Engineering College of Armed Police Force, Xi'an
710086, Shaanxi, China

a192liu@163.com

Keywords: tire; fuzzy; design

Abstract: This paper designed the safe driving speed analytical system on the basis of Matlab auxiliary fuzzy control, builds its model of the multi-sensors information fusion. According to the real time monitored temperature and pressure, applying fuzzy control technology, determining of the driving speed security situation is the one of required function of the tire system for the explosion, it is the key to reflect the system's level of intelligence. It has a very profound significance on eliminating the unsafe hidden danger of flat tire fundamentally. According to the real time monitored temperature and pressure, applying fuzzy control technology, determining of the driving speed security situation is the one of required function of the tire system for the explosion, it is the key to reflect the system's level of intelligence.

Car tire is an important part of the car, the performance of the tire will have direct influence on safety, braking performance, driving comfort, stability, cross - country property and fuel economy.[1] However, car tires' long-term exposure to external environment, direct contact with the ground, keeping a high speed and big load in car high-speed process are very easy to create the tire overpressure, overheating, under-pressure, a kind of any situation occur will cause the tire instantaneous blast. The best way to solve the problem above is a set of explosion-proof warning system which can monitor the temperature and the pressure of tires for the real time. At present, there is no practical tire explosion-proof product in the domestic. In the product, the system only monitors the tires' temperature and pressure, alarm automatically to tire overpressure, overheating and under-pressure.[2] This article utilizes the fuzzy control theory, makes safety analysis on the traffic speed according to the temperature and pressure which are monitored. It determines the safety status of speed and membership degrees and eliminates the tires of tire security risks fundamentally.

\section{Design of Fuzzy Controller}

In this system, at the conclusion of the manual control strategy, we use the Matlab Aid fuzzy controller auxiliary, in order to achieve the fuzzy speed safety analysis scheme of the whole system.

1.1 Linguistic Variables of the Fuzzy Controller

The input linguistic variables of Fuzzy controller may be the tire temperature and pressure, and output linguistic variables may be the speed. So, a double input-single output fuzzy controller for driving speed safety analysis of fuzzy control system is selected.

1.2 Determine the Basic Universe and Linguistic Value Theory of Linguistic Variables

This paper sets the type 185/70SR13 tires for research object, sets the basic universe of pressure $F$ for [0,300]. Select 3 linguistic values for linguistic variable F: PB, P0, NB; The basic universe of the temperature $\mathrm{T}$ is $[0,160]$. Selects 3 linguistic values for linguistic variable T: PB, PM, P0; The basic universe of speed $v$ is $[0,175]$. Select 4 linguistic values for linguistic variables: PB, PM, P0, NS. Membership functions are in chart 1,2, 3. 


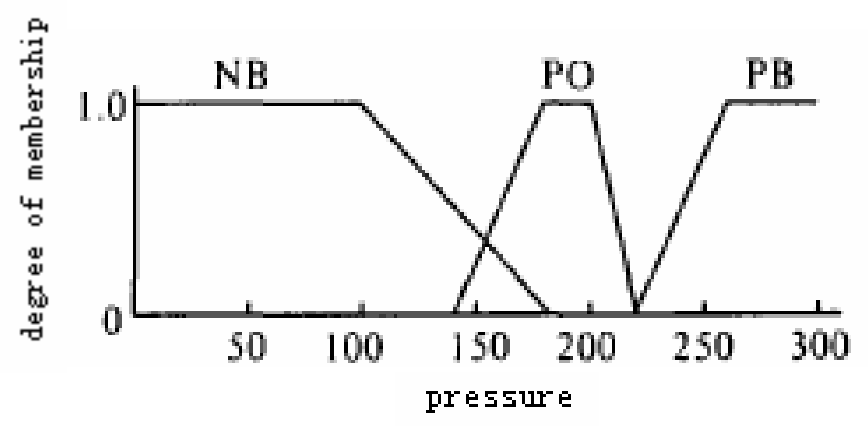

Figure 1 pressure membership functions

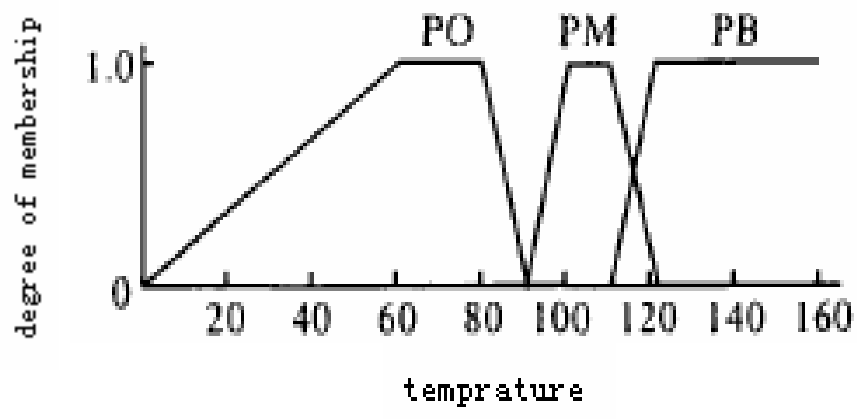

Figure 2 temperature membership functions

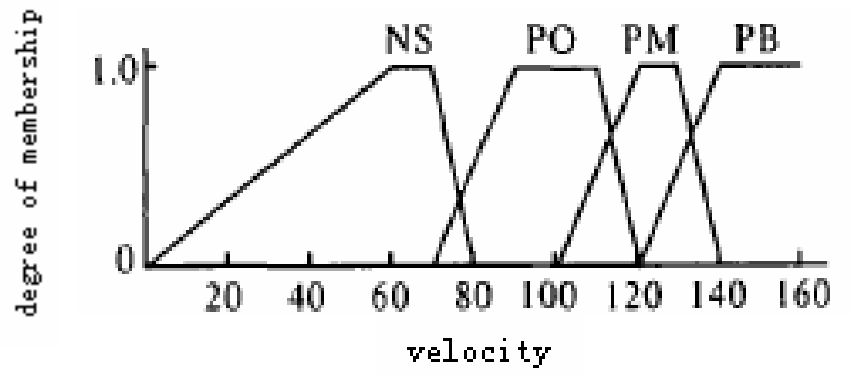

Figure 3 speed membership functions

\subsection{Fuzzy Control Status Table}

Based on the conclusion of the operator's manual control strategy, it summarizes a group of 9 control rules formed by fuzzy conditional statement.

(1) if $\mathrm{F}=\mathrm{PB}$ and $\mathrm{T}=\mathrm{PM}$ then $\mathrm{V}=\mathrm{PB}$;

(2) or if $\mathrm{F}=\mathrm{PB}$ and $\mathrm{T}=\mathrm{PB}$ then $\mathrm{v}=\mathrm{PB}$;

(3) or if $\mathrm{F}=\mathrm{P} 0$ and $\mathrm{T}=\mathrm{P} 0$ then $\mathrm{v}=\mathrm{P} 0$ :

(4) or $\quad \ldots \quad \ldots \quad \ldots$;

(8) or $\quad \ldots \quad \ldots \quad \ldots$;

(9) or if $\mathrm{T}=\mathrm{PB}$ then $\mathrm{v}=\mathrm{PB}$.

Summarizing these 9 fuzzy conditional statements above, it can build the fuzzy control status table of traffic speed safety analysis fuzzy control system control rules (table 1), " $\times$ " in the table, called a dead zone, stands for conditions what may not happen.

In this fuzzy controller, the synthesis order of each rule's result uses the method of maximum Max. Blurring method uses the area center method. [3] 
Table 1 fuzzy control status table

\begin{tabular}{ccccc}
\hline T & NB & P0 & PB & NONE \\
\hline P0 & NS & P0 & $\times$ & $\times$ \\
PM & PM & PM & PM & PM \\
PB & PB & $\times$ & PB & PB \\
\hline
\end{tabular}

\section{Control System Modeling and Simulation}

\subsection{Physical Model and Mathematical Model}

2.1.1 Fuzzy Controller There isn't a precise mathematical formula between the real-time monitoring of tires' temperature, pressure and cars' speed. Therefore, this system uses fuzzy controller to achieve the relationship of these 3 items.

2.1.2 Speed Manipulation of the Agency's Position Converter Speed manipulation of the agency's position converter is the input part of system, its purpose is to convert the position transformation of the speed manipulated mechanism to the corresponding speed, the mathematical relationship between them are as follows:

$$
\mathrm{v}=50 \mathrm{x}+45
$$

$\mathrm{x}$ is the position of speed control mechanism, $\mathrm{v}$ is the corresponding speed.

2.1.3 The Discrete Driving Controller Driving controller is the core of the system. Simply said, its function is producing the corresponding traction based on the current speed and specified speed difference. Driving controller is a typical PID controller. The mathematical description is

Integral link: $x(n)=x(n-1)+u(n)$,

Differential link: $d(n)=u(n)-u(n-1)$,

The system output: y $(\mathrm{n})=\mathrm{Pu}(\mathrm{n})+\mathrm{Ix}(\mathrm{n})+\operatorname{Dd}(\mathrm{n})$,

In the description, $\mathrm{u}(\mathrm{n})$ is the system input, equivalent to the difference between current speed and the designated speed. $y(n)$ is the system output, equivalent to car traction; $x(n)$ is the system state. P, I and D are the proportion of PID controller, integral and differential control parameters, their values are $\mathrm{P}=1, \mathrm{I}=0.01, \mathrm{D}=0$.

2.1.4 Car Dynamic Mechanism Car dynamic mechanism is the actuator of system. Its function is to change the motor vehicle speed under the action of the traction, take it to a specified speed. The relationship between the traction and the speed is

$$
\mathrm{F}=\mathrm{mv}+\mathrm{bv}
$$

$\mathrm{v}$ is the speed of the car, $\mathrm{F}$ is traction of car, $\mathrm{m}=1000 \mathrm{~kg}$ is the quality of the car, $\mathrm{b}=20$ is resistance factor.

\subsection{Establish Simulation Model}

According to the physical model and mathematical description of the system, establish the system simulation model in simulink simulation environment (figure 4). [4]

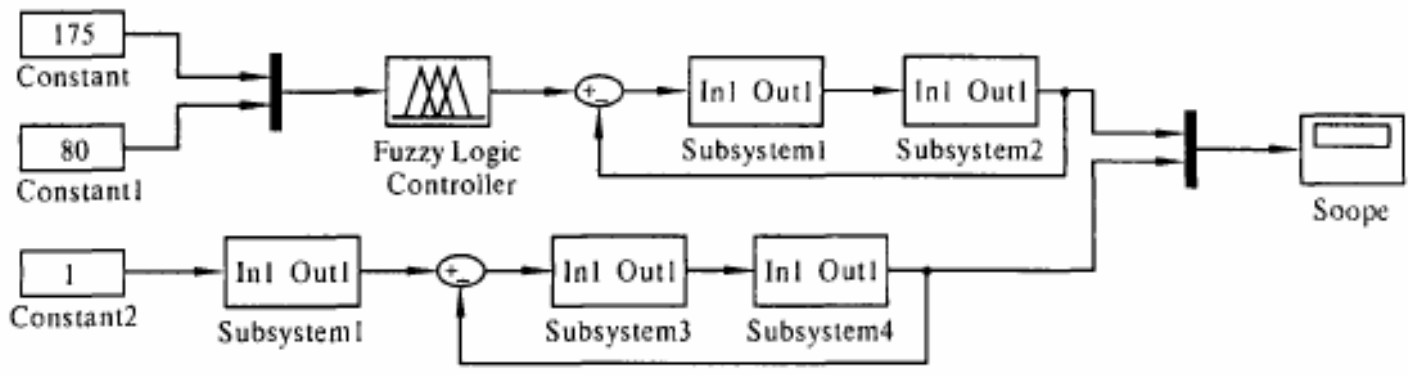

Figure 4 system simulation model 
Fuzzy Logic Controller, Subsystem1, Subsystem2, Subsystem3, Subsystem4 are packaged son modules.

\subsection{System Simulation and Analysis}

Use toolbox of Fuzzy Matlab6.5 to design Fuzzy system, compiled in Fuzzy window input and output variables of the membership function setting, in the Fuzzy rules editor set the rules, and other area center method for the solution of Fuzzy, finally using simulink tool of the Fuzzy on Fuzzy Controller components will have been set up two input and single output Mamdani of Fuzzy control system was charged into simulink system, and was charged with seamless connection system, and finally realize to the system, and simulation for simulation results. Structure of Simulink is shown in figure 5. [5]

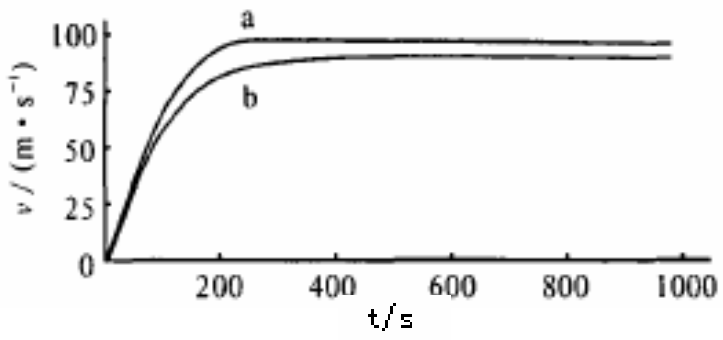

Figure 5 Comparison between speed decided by driving controller and the speed obtained by the fuzzy controller
a. driving controller
b. fuzzy controller

From the simulation results that the driving controller of speed and decided by the speed of the fuzzy controller that, after $200 \mathrm{~s}$ after step response is identical. [6]

\section{Conclusions}

According to the real time monitored temperature and pressure, applying fuzzy control technology, determining of the driving speed security situation is the one of required function of the tire system for the explosion, it is the key to reflect the system's level of intelligence. [7] It has a very profound significance on eliminating the unsafe hidden danger of flat tire fundamentally.

\section{References}

[1] Jeff B. Tire pressure monitoring: an industry under pressure [J] .Sensors (Peterborough, NH), 2003, 20 (7):29-33.

[2] Philips Semiconductors. Preliminary Specification of PCH7970 Pressure Sensor Signal Conditioning Chip [P] . 2003.

[3] GE NovaSensor. GE Nova Sensor NPX series sensor [P]. 2004.

[4] Alfred Pohl, Member, IEEE, Reinhard Steindl, and Leonhard Reindl, Member, IEEE. The "Intelligent Tire" Utilizing Passive SAW Sensors-Measurement of Tire Friction.

[5] Hyok J·Song, HuiP·Hsu, Richard Wiese. Modeling Signal Strength Range of TPMS in Automobiles.

[6] Wang Jun, Su Jianbo, Xi Yugeng, COM-based Software Architecture for Multi-sensor Fusion System[J], Information Fusion, 2001 ,2(4):261-2701

[7] Gao JB, Harris CJ, Some remarks on Kalman filters for the multi-sensor Fusion[J], Information Fusion, 2002, 3:191-2011 